\title{
Análise dos efeitos de perguntas e de instruções sobre o comportamento não-verbal ${ }^{1}$
}

\author{
Mariella Vasconcelos Nogueira Braga \\ Luiz Carlos de Albuquerque \\ Carla Cristina Paiva Paracampo \\ Universidade Federal do Pará
}

\begin{abstract}
RESUMO
Este estudo investigou, com 8 universitários, se perguntas podem controlar comportamento tal como instruções. Utilizou-se um procedimento de escolha segundo o modelo, com três estímulos de comparação; a tarefa consistia em apontar para cada um dos três estímulos de comparação, em sequiência. As sequiências corretas eram reforçadas em FR6. Na Condição I, as Fases 1 (linha de base), 2, 3, 4 e 5 eram iniciadas com apresentação de instrução mínima, instrução correspondente, arranjo de estímulos, pergunta e arranjo de estímulos, respectivamente. Na Condição II foi invertida a ordem da apresentação da instrução correspondente e da pergunta. A instrução correspondente e a pergunta descreviam corretamente as contingências nas fases em que eram apresentadas. As transições entre as Fases 2, 3, 4 e 5 também eram marcadas pela mudança nas contingências. Observou-se que perguntas que especificam o comportamento que produz reforço, tal como instruções que especificam esse comportamento, podem estabelecer comportamentos novos e gerar insensibilidade do comportamento a mudanças nas contingências de reforço programadas. Sugere-se que tais perguntas, assim como instruções, podem ser classificadas como regras. Mas o controle exercido por instruções tende a apresentar pouca variação e a ser mais persistente quando comparado com o controle exercido por perguntas.
\end{abstract}

Palavras-chave: definição de regras; controle instrucional; controle por perguntas.

\begin{abstract}
Analysis of the effects of questions and of the instructions on nonverbal behavior

This study was conducted with eight university students and sought to ascertain whether questions, like instructions, might also control behavior. A matching-to-sample procedure with three comparison stimuli was used. Each participant pointed to the three comparisons in a specific sequence. The correct responses were reinforced on an FR6 schedule. In Condition 1, Phases 1 (baseline), 2, 3, 4 and 5 began with the following type of instruction: minimal, correspondent, stimulus arrangement, and questioning and stimulus arrangement. In Condition II, the order of correspondent instructions and questioning were reversed. The correspondent and questioning instruction correctly described the contingencies in those phases where they were present. In addition, in the transition between Phases 2 , 3,4 and 5, a contingency change was introduced. It was observed that those questions that produced behavioral reinforcement resulted in new behaviors and generated behavioral insensitivity to changes in programmed reinforcement contingencies. This finding suggests that, similar to instructions, questions may be considered as rules. However, in contrast to behavior control by questions, instructional control tended to be less variable and more consistent in its effects.
\end{abstract}

Keywords: rule definition; instructional control; control by questioning.

De acordo Skinner (1957) comportamento verbal é o comportamento que não age diretamente sobre o mundo físico e é reforçado por um ouvinte treinado de acordo com as práticas do grupo do qual o ouvinte e o falante são membros. Estímulos verbais, ainda de acordo Skinner, são os produtos do comportamento verbal. Skinner (1969) classificou como regras os estímulos verbais que especificam contingências, isto é, que especificam as relações entre os eventos que antecedem o comportamento, o próprio comportamento e suas prováveis conseqüências. Tais estímulos verbais funcionariam como estímulos discriminativos fazendo parte de um conjunto de contingências de reforço. Por essa definição, instruções, avisos, orientações, conselhos, ordens, leis etc., seriam exemplos particulares de regras, uma vez que todos podem descrever contingências e funcionar como estímulos discriminativos.

Zettle e Hayes (1982) concordam que regras são estímulos antecedentes verbais. Estímulo verbal, de acordo com Hayes (1986), "é aquele que tem seus efeitos discriminativo, estabelecedor, eliciador, reforçador, ou outros, devido a sua participação em quadros relacionais estabelecidos por uma comunidade 
verbal" (p. 357). Por essa visão, estímulos verbais e não-verbais podem ter as mesmas funções, uma vez que estímulos não-verbais também podem funcionar como discriminativo, estabelecedor, eliciador e reforçador. A diferença estaria no processo através do qual estes estímulos adquiririam as suas funções. Assim, em uma classe de estímulos equivalentes, quando um membro adquire uma função (discriminativa, por exemplo), todos os demais membros da classe também adquirem a função. Ou seja, enquanto estímulos discriminativos não-verbais adquirem essa função devido a uma história de reforço diferencial, estímulos discriminativos verbais a adquirem por causa de sua participação em quadros relacionais ou classes de equivalência (Hayes, 1986; Hayes \& Hayes, 1989). Zettle e Hayes argumentam que a visão de regras como antecedentes verbais tem as vantagens de evitar o problema do que significa especificar contingências e incorporar regras que não especificam claramente contingências.

Por essa definição de regras proposta por Zettle e Hayes (1982), portanto, qualquer estímulo antecedente verbal poderia funcionar como regra, independentemente de sua forma. Assim, se for considerado que a principal função de regras é a de substituir as contingências de reforço no estabelecimento de comportamentos novos (Catania, 1998), deveria ser esperado que qualquer estímulo antecedente verbal, independentemente de sua forma, pudesse exercer essa função. Deveria ser esperado, por exemplo, que perguntas, enquanto estímulos antecedentes verbais, também pudessem estabelecer comportamentos novos, antes mesmo destes comportamentos manterem contato com as suas conseqüências imediatas. Isso considerando que o comportamento de seguir regra, por definição, ocorreria independentemente das consequiências imediatas por ele produzidas (Albuquerque, Matos, de Souza \& Paracampo, 2004).

De acordo com Skinner (1969) perguntas podem gerar o comportamento de auto-observação. Ou seja, perguntas, tais como: “O que você fez?", "O que você está fazendo?”, e “O que você vai fazer?”, podem contribuir para tornar o indivíduo consciente das variáveis que controlam o seu comportamento. Além disso, podem levar o indivíduo a formular regras que poderão vir a afetar o seu comportamento não-verbal subsequiente. Essas proposições de Skinner têm sido abordadas em alguns estudos na linha de pesquisa que investiga o controle por regras (Bentall \& Lowe, 1987; Catania, Matthews \& Shimoff, 1982; Catania, Shimoff \& Matthews, 1989; Cerutti, 1991; Dixon \& Hayes, 1998; Harzem, Lowe \& Bagshaw, 1978; LeFrancois, Chase \& Joyce, 1988; Lippman \& Meyer, 1967; Lowe, 1979; Paracampo, de Souza, Matos \& Albuquerque, 2001; Pouthas, Droit, Jacquet \& Wearden, 1990; Rosenfarb, Newland, Brannon \& Howey, 1992;
Torgrud \& Holborn, 1990; Vaughan, 1985). Tais estudos têm contribuído tanto para identificar as condições sob as quais as verbalizações dos participantes podem interferir em seus desempenhos não-verbais, quanto para estabelecer as condições necessárias para que se possa dizer que tais verbalizações exerceram controle sobre desempenhos não-verbais.

Um problema, no entanto, é que nessa linha de pesquisa poucos estudos têm sido planejados com o objetivo de investigar os efeitos de perguntas sobre o comportamento não-verbal. Em alguns desses estudos (Catania \& cols., 1982; Catania \& cols., 1989; Cerutti, 1991; Harzem \& cols., 1978; LeFrancois \& cols., 1988; Lippman \& Meyer, 1967; Lowe, 1979; Paracampo \& cols., 2001; Rosenfarb e cols., 1992; Torgrud \& Holborn, 1990) os participantes têm sido solicitados, seja através de perguntas, seja através de pedidos, a descreverem as contingências a que são expostos, mas esses estudos não têm procurado investigar os efeitos de tais estímulos verbais sobre o comportamento não-verbal. Ao invés, têm procurado avaliar os efeitos das respostas verbais (descrições das contingências) às perguntas ou aos pedidos sobre o comportamento não-verbal. Deste modo, tais estímulos verbais têm sido utilizados mais como instrumentos de coleta de dados do que como variável controladora do comportamento não-verbal.

Um dos estudos que analisou os efeitos de estímulos antecedentes verbais (no caso, pedidos) tanto sobre o comportamento verbal quanto sobre o não-verbal, foi o conduzido por Simonassi, Oliveira e Gosch (1997). No Experimento I desse estudo, 20 universitários foram distribuídos em duas condições experimentais. Nas duas condições a tarefa dos participantes era colocar um de vários cartões numerados em uma de duas caixas, uma de cor verde e outra, vermelha. As respostas corretas eram colocar cartões com números pares na caixa verde e cartões com números ímpares na caixa vermelha. Tais respostas eram conseqüenciadas com "certo". Já as incorretas e, portanto, conseqüenciadas com "errado", eram colocar cartões com números pares na caixa vermelha e cartões com números ímpares na caixa verde. Uma tentativa foi definida como a resposta de colocar um cartão em uma das caixas, seguida por sua conseqüência. Em alguns intervalos entre tentativas, o participante era solicitado, através de um pedido, a descrever como estava resolvendo o problema. A participação do estudante no experimento era encerrada de acordo com um dos seguintes critérios, o que ocorresse primeiro: 1) após a emissão de 25 respostas corretas consecutivas ou 2) após a ocorrência de 520 tentativas. As duas condições diferiram apenas quanto à especificidade do pedido. Na Condição I, o pedido era "Escreva nesta folha como você está distribuindo os cartões com números em relação às caixas verde e vermelha". Já 
na Condição II, era "Escreva nesta folha como você está fazendo para resolver este exercício”. Dezoito participantes atingiram e dois não atingiram o critério de desempenho para o encerramento do experimento. Desses 18 participantes, 16 (oito de cada condição) atingiram esse critério até a tentativa 105. Oito participantes da Condição I e seis da Condição II, formularam a regra. Desses, cinco da Condição I e um da Condição II formularam a regra até a tentativa 3, e 13 apresentaram redações de palavras que descreveram os termos da contingência antes de a regra ser formulada. De acordo com os autores, a solução do problema dependeu das contingências de reforço programadas nas duas condições, enquanto que a formulação da regra foi afetada pela exposição às contingências e pelo conteúdo do pedido na Condição I, e pela exposição às contingências na Condição II. Isto considerando que, na Condição I, um maior número de participantes formulou a regra até a terceira tentativa e que os participantes dessa condição usaram com maior freqüência, nas regras por eles formuladas, as palavras que faziam parte do pedido.

Nessa mesma linha de investigação, Silva e Albuquerque (submetido) avaliaram os efeitos de perguntas sobre o comportamento não-verbal. Neste estudo, nove estudantes universitários foram expostos a um procedimento de escolha segundo o modelo. Em cada tentativa, um estímulo modelo e três de comparação eram apresentados ao participante e, em seguida, uma lâmpada era acesa. Na presença desses estímulos, o participante deveria apontar para os três estímulos de comparação em uma dada sequiência. As seqüências corretas eram reforçadas (com pontos trocáveis por dinheiro) em CRF. O experimento era constituído de três condições e cada condição de quatro sessões. As contingências na Sessão 1 eram alteradas na Sessão 2, restabelecidas na Sessão 3 e mantidas inalteradas na Sessão 4, que era iniciada com a apresentação da regra discrepante das contingências. Durante a Condição I, os participantes não eram solicitados a verbalizar e durante as Condições II e III, eram solicitados, através de perguntas, a descreverem o comportamento não-verbal que produzia reforço. Na Condição II, as perguntas eram as seguintes: “Quando a lâmpada da esquerda estiver acesa, o que você deve fazer?"; e "Quando a lâmpada da direita estiver acesa, o que você deve fazer?”. Já na Condição III, eram as seguintes: "Quando a lâmpada da esquerda estiver acesa, você deve apontar para os objetos de comparação em que seqüência para ganhar pontos?"; e "Quando a lâmpada da direita estiver acesa, você deve apontar para os objetos de comparação em que sequiência para ganhar pontos?". As respostas verbais às perguntas nunca eram reforçadas. A Sessão 1 era encerrada após a emissão de dez seqüências de respostas não-verbais corretas consecutivas. Caso o participante não atingisse esse critério em 120 tentativas, a sua participação no experimento era encerrada na Sessão 1. Assim, só eram expostos às Sessões 2, 3 e 4 os participantes que atingissem o critério de encerramento da Sessão 1. As Sessões 2, 3 e 4 eram encerradas obedecendo a um dos seguintes critérios, o que ocorresse primeiro: a) a emissão de 10 seqüências de respostas não-verbais corretas consecutivas; ou, b) a ocorrência de 80 tentativas. Os três participantes da Condição I não apresentaram um desempenho discriminado, de acordo com as contingências de reforço na Sessão 1. Já dois participantes da Condição II e os três da Condição III, apresentaram desempenhos não-verbal e verbal de acordo com as contingências na Sessão 1 e sensíveis às mudanças nas contingências nas Sessões 2 e 3 . Desses cinco, quatro deixaram de seguir a regra discrepante na Sessão 4.

De acordo com Silva e Albuquerque (submetido), os resultados sugerem que solicitar ao participante, através de perguntas, que descreva o comportamento que produz reforço ao longo de sua exposição às contingências pode facilitar a aquisição de discriminações condicionais. A forma dos comportamentos apresentados na Sessão 1, pelos participantes das Condições II e III, não foi determinada exclusivamente pelas perguntas, porque elas não especificavam quais respostas verbais deveriam ser apresentadas, nem especificavam quais seqüências de respostas nãoverbais deveriam ser emitidas na presença dos estímulos de comparação, quando as lâmpadas eram acesas. Além disso, nenhum dos participantes dessas duas condições passou a responder corretamente imediatamente após a primeira apresentação das perguntas. As perguntas, no entanto, podem ter funcionado como uma regra geral, indicando que os participantes deveriam apontar para os estímulos de comparação em sequiência. No caso da Condição III, as perguntas indicaram mais que isso. Nesta condição elas especificaram o comportamento (apontar em seqüência), a situação na qual ele deveria ocorrer (na presença dos estímulos de comparação, quando uma lâmpada fosse acesa) e suas consequiências (obtenção de pontos). Deste modo, as perguntas, principalmente na Condição III, podem ter restringido a variabilidade do comportamento e, em conseqüência, facilitado o controle pelas contingências de reforço no estabelecimento da tarefa de discriminação condicional.

Essa análise sugere que uma das exigências para que se possa dizer que um estímulo antecedente verbal determinou um comportamento, é que tal comportamento seja previamente especificado por esse estímulo. Se for assim, uma questão que surge, então, é se basta que um estímulo antecedente verbal especifique um comportamento para que esse comportamento ocorra ou esse comportamento ocorrerá ou não, dependendo de outros fatores. Em relação a essa ques- 
tão, há evidências experimentais mostrando que esse comportamento pode ocorrer ou não, dependendo, em parte, da complexidade do próprio estímulo antecedente verbal que o especifica, isto é, dependendo da extensão desse estímulo, medida pelo número de diferentes respostas por ele especificadas (Albuquerque \& Ferreira, 2001). O que ainda não está claro, no entanto, é se, além da complexidade, outras propriedades formais de tais estímulos (isto é, dos estímulos antecedentes verbais que especificam o comportamento que produz reforço), como por exemplo, se eles são apresentados ao ouvinte na forma interrogativa (pergunta) ou na forma imperativa (instrução), também podem interferir na ocorrência do comportamento por eles especificado. Também não está claro se estas propriedades formais de tais estímulos podem interferir na sensibilidade ${ }^{2}$ do comportamento a mudanças nas contingências. Em outras palavras, não está claro se perguntas, tal como algumas outras formas de estímulos antecedentes verbais, também podem exercer a função de estabelecer comportamentos novos e gerar insensibilidade do comportamento a mudanças nas contingências, uma vez que poucos estudos têm procurado investigar experimentalmente essas questões.

Considerando esta análise, o presente estudo procurou investigar se uma pergunta é mais ou é menos provável de ser seguida do que uma instrução quando: 1) a pergunta e a instrução especificam o comportamento não-verbal que produz reforço; 2) esse comportamento é reforçado em FR 6; e, 3) a sua sensibilidade a mudanças nas contingências de reforço é testada.

Para tanto, foi utilizado um procedimento de escolha de acordo com o modelo, similar ao que tem sido usado por Albuquerque e colaboradores (Albuquerque \& Ferreira, 2001; Albuquerque \& cols., 2004), analisando-se os efeitos do procedimento em cada indivíduo independentemente. Esse procedimento foi utilizado porque permite avaliar, a cada tentativa, se as respostas não-verbais emitidas pelo participante se alternam ordenadamente entre as dimensões dos estímulos de comparação, de acordo com os estímulos antecedentes verbais ou com as contingências de reforço programadas.

\section{MÉTODO}

\section{Participantes}

Os participantes foram oito estudantes universitários sem história experimental prévia, matriculados em diferentes cursos (exceto o de Psicologia) e diferentes semestres. Todos foram convidados a participar do experimento como se segue:

"Estou realizando uma pesquisa sobre aprendizagem e queria saber se você estaria interessado em participar. O objetivo da pesquisa é investigar processos de aprendizagem comuns a todas as pessoas e será realizada no laboratório de Psicologia, aqui da universidade. A pesquisa será realizada em quatro dias da semana, durante uma hora por dia, aproximadamente. A pessoa que participar receberá a passagem de ônibus de ida e volta à universidade. Além disso, poderá receber mais uma quantia em dinheiro no final da pesquisa. Você está interessado em participar?"

\section{Equipamentos e material}

Foi utilizada uma mesa de madeira, medindo $150 \mathrm{x}$ 78 x $70 \mathrm{~cm}$. Fixado à mesa, de modo a dividi-la ao meio em todo o seu comprimento, havia um anteparo com espelho unidirecional de 150 x $60 \mathrm{~cm}$, fixado em uma moldura de madeira e localizado $13 \mathrm{~cm}$ acima do tampo da mesa. No centro do anteparo, junto ao tampo da mesa, havia uma abertura retangular de $45 \times 3$ $\mathrm{cm}$. Acima e ao centro dessa abertura havia um contador operado pelo experimentador e com os dígitos voltados para o participante. Visível ao participante estava instalada no anteparo uma lâmpada transparente de 5 watts com uma etiqueta de papel com a frase impressa: "Você ganhou um ponto". Uma lâmpada fluorescente de 15 watts estava instalada na borda superior e ao centro do anteparo. Ao lado direito do experimentador, havia duas fitas cassetes, um amplificador e um tape-deck. Conectados ao tape-deck, havia dois fones de ouvido. A mesa estava situada no centro de uma sala refrigerada por um condicionador de ar.

Os estímulos modelo e de comparação eram peças de madeira (blocos lógicos da marca Funbec), variando em três dimensões: forma (quadrado, círculo, retângulo e triângulo), cor (azul, vermelha e amarela) e espessura (grossa e fina). Estas peças de madeira formavam 40 diferentes arranjos de estímulos, cada um constituído de um estímulo modelo e três estímulos de comparação. Cada estímulo de comparação apresentava apenas uma dimensão (cor, espessura ou forma) em comum com o estímulo modelo e diferia nas demais. A combinação dos estímulos era aleatória, assim como a ordem de apresentação dos 40 arranjos. Os arranjos de estímulos, previamente preparados, ficavam sobre a mesa, ao lado do experimentador, na ordem em que seriam apresentados em cada tentativa. Para facilitar o manejo dos arranjos, sentado ao lado esquerdo do experimentador, um auxiliar de pesquisa aproximava os arranjos, conforme os mesmos iam sendo apresentados. Os estímulos eram apresentados ao participante através da abertura na base do anteparo divisor da mesa, em uma bandeja de madeira em forma de ' $T$ '. Na parte final do cabo dessa bandeja, rente à base retangular, quatro ripas de madeira formavam um quadrado no qual era colocado o estímulo modelo. 
$\mathrm{Na}$ base retangular, dividida por ripas de madeira em três quadrados, eram apresentados os três estímulos de comparação. As respostas não-verbais (apontar para os estímulos de comparação) emitidas pelos participantes eram registradas pelo experimentador em um protocolo previamente preparado e eram também gravadas pela filmadora, para análises posteriores. Os reforçadores utilizados eram pontos, registrados no contador, que eram trocados por dinheiro ao final do experimento. Cada ponto valia $\mathrm{R} \$ 0,20$ (vinte centavos de real).

\section{Procedimento}

Durante as sessões experimentais, participante e experimentador ficavam sentados à mesa, de frente um para o outro, separados pelo anteparo divisor da mesa. A lâmpada na borda superior do anteparo ficava constantemente acesa, voltada para o participante, de maneira a assegurar que seu lado apresentasse iluminação em maior intensidade, garantindo que apenas as ações emitidas pelo participante, bem como o arranjo de estímulos apresentado, pudessem ser observados através do espelho. $\mathrm{O}$ experimentador, em algumas sessões, inicialmente apresentava ao participante uma determinada instrução ou uma pergunta e em seguida apresentava os arranjos de estímulos; em outras, só apresentava os arranjos de estímulos. As sessões duravam em média 30 minutos e o intervalo entre sessões era de aproximadamente 5 minutos. Em cada tentativa, o experimentador apresentava um dos 40 arranjos de estímulos. Na presença destes estímulos, o participante deveria apontar para cada um dos três estímulos de comparação em uma dada sequiência. Caso a sequiência de respostas emitida estivesse de acordo com as contingências de reforço programadas (sequiência correta), um ponto era acrescentado no contador, a lâmpada transparente era acesa e apagada e a bandeja com o arranjo de estímulos era retirada. Caso a sequiência de respostas fosse incorreta, a lâmpada transparente não era acesa e a bandeja com o arranjo de estímulos era retirada, sem que fosse acrescentado um ponto no contador. Havia um intervalo variável de aproximadamente 5 segundos entre uma tentativa e outra.

Os pontos eram registrados cumulativamente, no contador, apenas dentro de uma mesma sessão. No entanto, a partir da terceira sessão, logo após entrar na sala experimental, o participante era informado pelo experimentador sobre o número total de pontos obtidos nas sessões anteriores.

\section{Orientações preliminares}

$\mathrm{Na}$ primeira sessão, quando participante e experimentador entravam na sala, a bandeja com um arranjo de estímulos estava sobre a mesa, portanto, visível ao participante. $\mathrm{O}$ experimentador pedia ao participante que se sentasse na cadeira e, ao lado do participante, sempre apontando com o dedo para cada um dos estímulos a que se referira, dizia: "Este objeto, aqui em cima, é um modelo. Estes três objetos, aqui em baixo, são para você comparar com o modelo. Nós vamos chamar estes três objetos, aqui em baixo, de objetos de comparação. Observe que cada um destes três objetos de comparação tem uma única propriedade comum ao modelo. [Veja. Este, só tem a espessura comum ao modelo. Este aqui, só tem a cor comum ao modelo. Este aqui só tem a forma igual ao modelo]. Quando eu apresentar os objetos de comparação para você, você deverá apontar com o dedo para cada um dos três objetos de comparação em uma dada seqüência. Fazendo isso você poderá ganhar pontos que serão trocados por dinheiro. Cada ponto que você ganhar será trocado por $\mathrm{R} \$ 0,20$ (vinte centavos), mas somente no final da pesquisa. Quando você ganhar pontos, os pontos sempre aparecerão aqui neste contador e esta lâmpada será acesa. Veja como os pontos aparecem no contador e como a lâmpada será acesa (o auxiliar, que se encontrava no outro lado da mesa, acionava o contador e acendia a lâmpada por cinco vezes). Quando você não ganhar pontos, nenhum ponto será acrescentado no contador e a lâmpada não será acesa. Entendeu?". Este procedimento era repetido duas vezes e ocorria apenas no início da primeira sessão. Na segunda vez em que essas orientações preliminares eram apresentadas, o trecho entre colchetes era omitido.

\section{Instruções e pergunta}

Logo após as orientações preliminares serem apresentadas ao participante, o experimentador pedia para o participante colocar os fones de ouvido e se deslocava em direção à sua cadeira. Separado do participante pelo anteparo com espelho unidirecional, o experimentador também colocava os seus fones de ouvido e entregava ao participante, pela abertura na base do anteparo, uma folha de papel contendo uma das seguintes instruções ou pergunta datilografada:

\section{Instrução mínima}

"Aponte com o dedo para os objetos de comparação".

\section{Instrução correspondente}

"Quando eu mostrar estes objetos para você, você deve fazer o seguinte: Você deve apontar primeiro para a mesma cor [forma], depois para a mesma espessura [cor] e em seguida para a mesma forma [espessura]". 


\section{Pergunta}

"Quando eu mostrar estes objetos para você, o que você deve fazer? Você deve apontar primeiro para a mesma cor [forma], depois para a mesma espessura [cor] e em seguida para a mesma forma [espessura]?".

\section{Delineamento experimental}

Os participantes foram distribuídos em duas condições experimentais. Cada condição, realizada com quatro participantes, era constituída de cinco fases. $\mathrm{Na}$ Condição I (IP), a Fase 1 (fase de linha de base) era iniciada com a apresentação da instrução mínima, a Fase 2 com a apresentação da instrução correspondente (I), a Fase 3 com a apresentação de um arranjo de estímulos, uma vez que não eram apresentadas instruções ou pergunta nesta fase, a Fase $4 \mathrm{com}$ a apresentação da pergunta (P), e a Fase 5 com a apresentação de um arranjo de estímulos, uma vez que também não eram apresentadas instruções ou pergunta nesta fase. Na Condição II (PI), a Fase 1 (fase de linha de base) era iniciada com a apresentação da instrução mínima, a Fase 2 com a apresentação da pergunta, a Fase 3 com a apresentação de um arranjo de estímulos, uma vez que não eram apresentadas instruções ou pergunta nesta fase, a Fase 4 com a apresentação da instrução correspondente, e a Fase 5 com a apresentação de um arranjo de estímulos, uma vez que também não eram apresentadas instruções ou pergunta nessa fase $^{3}$. Nas duas condições, a Fase 1 era encerrada após 10 tentativas serem apresentadas e cada uma das Fases 2, 3, 4 e 5 era encerrada após duas sessões serem completadas. Cada sessão, a partir da Fase 2, inclusive, era constituída de 80 tentativas.

Nas duas condições, a Fase 1 era constituída de única sessão de linha de base em relação à qual eram avaliados os efeitos da introdução da instrução correspondente na Fase 2 da Condição I (IP), e da introdução da pergunta na Fase 2 da Condição II (PI). Durante a Fase 1 nenhuma resposta era reforçada. Durante a Fase 2 era reforçada apenas uma de duas seqüências de repostas: a seqüência cor - espessura forma (CEF) ou a sequiência FCE. Qual destas duas sequiências era reforçada, dependia do desempenho do participante na Fase 1. Se na Fase 1 o participante emitisse a sequiência CEF em mais de $50 \%$ das tentativas, a sequiência reforçada na Fase 2 seria a seqüência FCE. Caso contrário, a sequiência reforçada na Fase 2 seria a sequiência CEF. Esse procedimento era usado para evitar coincidência entre o comportamento apresentado pelo participante na Fase 1 e o seu comportamento apresentado na Fase 2. A única seqüência de respostas reforçada durante a Fase 3 era a sequiência EFC; durante a Fase 4 era a sequiência FEC; e durante a Fase 5 era a seqüência ECF. Em cada uma das Fases $2,3,4$ e 5 quando uma seqüência de repostas era re- forçada, era reforçada em esquema de razão fixa 6 (FR 6). Nesse esquema de razão fixa, cada seis emissões consecutivas de uma mesma sequiência correta produzia um ponto. Erros ou a não emissão consecutiva de uma mesma sequiência correta, reiniciavam a razão fixa -6 para obtenção de um ponto.

$\mathrm{Na}$ Condição I (IP), a seqüência correta (programada para ser reforçada) na Fase 2 era previamente especificada pela instrução correspondente no início das Sessões 1 e 2 dessa fase; e a sequiência correta na Fase 4 era previamente especificada pela pergunta no início das Sessões 1 e 2 dessa fase. Na Condição II (PI), a sequiência correta na Fase 2 era previamente especificada pela pergunta no início das Sessões 1 e 2 dessa fase; e a seqüência correta na Fase 4 era previamente especificada pela instrução correspondente no início das Sessões 1 e 2 dessa fase. Nas duas condições, as seqüências corretas nas Fases 3 e 5 não eram especificadas pela instrução correspondente e nem pela pergunta. Nas duas condições, então, as transições da Fase 2 para a Fase 3 e da Fase 4 para 5 eram marcadas pela mudança não sinalizada nas contingências de reforço programadas.

\section{Forma de apresentação das instruções e pergunta}

Imediatamente após o participante receber a folha de papel contendo uma das instruções ou a pergunta datilografada, o experimentador ligava o tape-deck e, através dos fones de ouvido, o participante passava a ouvir uma fita, previamente gravada, que dizia: "Eu vou ler (dependendo da fase experimental, a gravação dizia: "esta instrução" ou "esta pergunta") para você em voz alta. Acompanhe minha leitura, lendo silenciosamente". A gravação continuava com a leitura da instrução ou da pergunta contida na folha de papel. Terminada essa primeira leitura, a gravação prosseguia: "Agora, você deve ler (dependendo da fase experimental, a gravação dizia: "esta instrução" ou "esta pergunta") sozinho, silenciosamente. Leia com calma e bastante atenção. Você tem todo o tempo que achar necessário para entendê-la. Quando você achar que entendeu bem (dependendo da fase experimental, a gravação dizia: "a instrução" ou "a pergunta"), avise-me". Terminada esta segunda leitura, a gravação prosseguia: "Devolva-me a folha com (dependendo da fase experimental, a gravação dizia "a instrução" ou "a pergunta"). Eu só posso falar com você agora, no início da próxima sessão. Você pode retirar os fones de ouvido agora". Este procedimento era utilizado no início de cada sessão das Fases 1, 2 e 4 das duas condições.

Logo após o participante devolver a folha, o experimentador removia a bandeja, voltava a apresentar a 
bandeja com um novo arranjo de estímulos, e dizia: "Comece a apontar".

\section{Comparação dos registros e término da participação do estudante no experimento}

Nas duas condições, a cada duas sessões, um observador independente comparava o registro feito pelo experimentador com o registro feito pela filmadora. Caso houvesse $100 \%$ de concordância entre os registros, o participante continuava participando do experimento. Caso contrário, o participante era descartado. No presente estudo nenhum participante foi descartado por essa razão. As sessões eram realizadas de segunda a sexta-feira e eram realizadas duas sessões, no máximo, por dia. A participação do estudante no experimento era encerrada quando o critério de encerramento da Fase 5 fosse atingido.
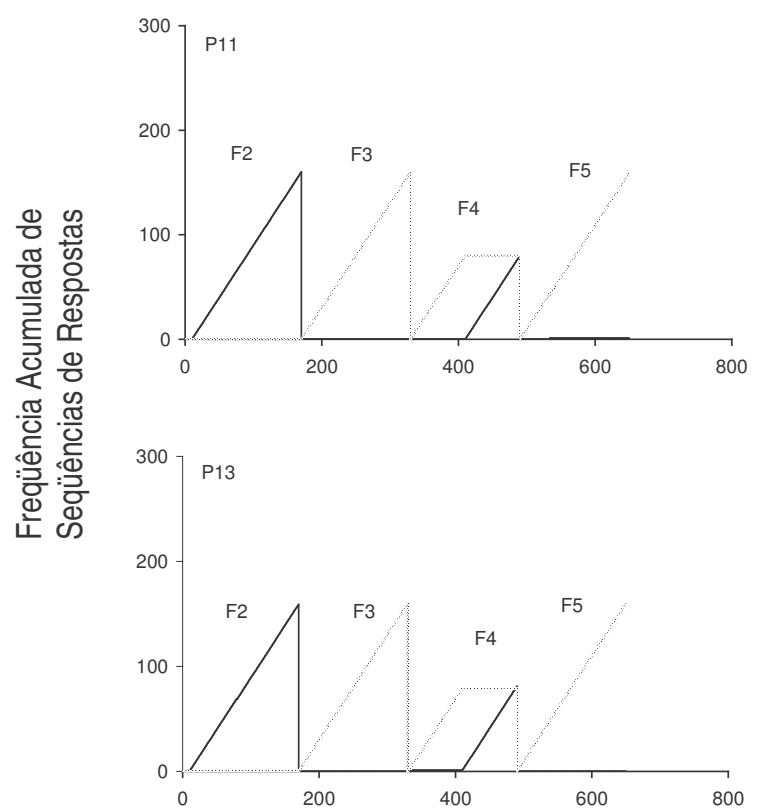

\section{RESULTADOS}

Durante a Fase 1 (linha de base) nenhum dos participantes das Condições I (IP) e II (PI) respondeu na sequiência $\mathrm{CEF}$ em mais de 50\% das tentativas. Desse modo, a Fase 2 da Condição I (IP) foi iniciada com a instrução correspondente especificando a seqüência CEF, e a Fase 2 da Condição II (PI) foi iniciada com a pergunta especificando a seqüência CEF.

\section{Condição I (IP)}

A Figura 1 mostra a frequiência acumulada de sequiências de respostas corretas e incorretas, emitidas por cada participante da Condição I (IP), durante as Fases 2, 3, 4 e 5. A Tabela 1 mostra as porcentagens de sequiências de respostas apresentadas durante as Fases 2, 3, 4 e 5, pelos participantes da Condição I (IP).
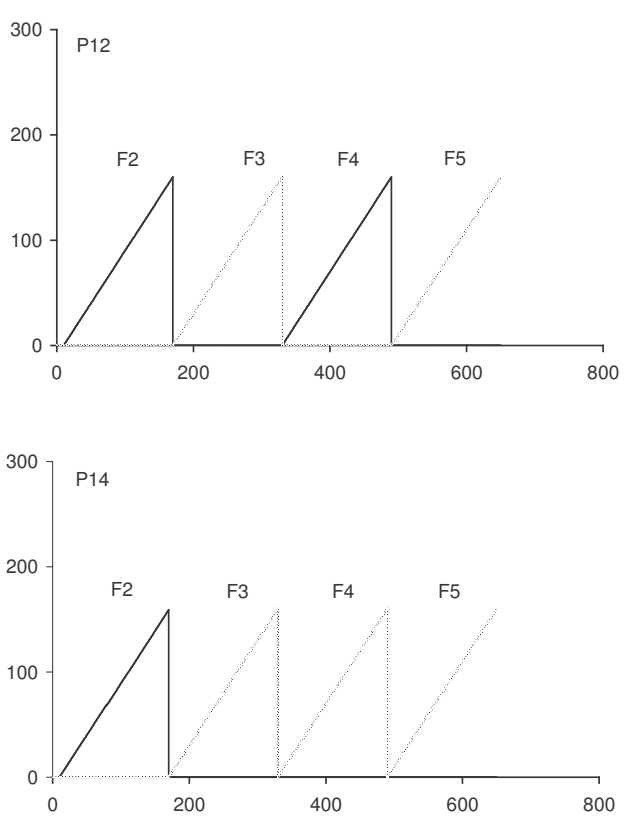

Tentativas

Figura 1: Freqüência acumulada de seqüências de respostas corretas (linha sólida preta) e incorretas (linha tracejada cinza), para cada participante (P), durante cada fase (F) experimental na Condição I (IP). Quebras na curva acumulada indicam mudanças de fase.

Na Fase 2 da Condição I (IP), quando a instrução correspondente às contingências foi introduzida, podese observar na Figura 1 que todos os participantes dessa condição (P11, P12, P13 e P14) iniciaram essa fase respondendo corretamente. Na Tabela 1 observase que esses quatro participantes responderam corretamente na seqüência CEF, especificada pela instrução correspondente, em mais de $99 \%$ das tentativas dessa fase.
Na Fase 3, quando as contingências foram alteradas e o seguimento de instrução deixou de produzir pontos, nenhum desses quatro participantes (P11, P12, P13 e P14) respondeu corretamente, de acordo com as novas contingências de reforço, como pode ser observado na Figura 1. Na Tabela 1 observa-se que todos esses quatro participantes continuaram emitindo a sequiência CEF, previamente especificada pela instrução correspondente, em mais de $97 \%$ das tentativas dessa fase. 
Tabela 1: Porcentagens de Seqüências de Respostas (Seq.) Emitidas pelos Participantes P11, P12, P13 e P14 da Condição I, Durante a Primeira e a Segunda Sessão de cada Fase, e Durante toda a Fase Experimental, nas Fases 2, 3, 4 e 5

\begin{tabular}{|c|c|c|c|c|c|c|c|c|c|c|c|c|}
\hline \multirow[b]{2}{*}{ Seq. } & \multicolumn{3}{|c|}{ Fase 2} & \multicolumn{3}{|c|}{ Fase 3} & \multicolumn{3}{|c|}{ Fase 4} & \multicolumn{3}{|c|}{ Fase 5} \\
\hline & Primeira & Segunda & Durante & Primeira & Segunda & Durante & Primeira & Segunda & Durante & Primeira & Segunda & Durante \\
\hline \multicolumn{13}{|c|}{ Participante P11 } \\
\hline $\mathrm{CEF}$ & 100 & 100 & 100 & 100 & 100 & 100 & 100 & 0 & 50 & 0 & 0 & 0 \\
\hline CFE & 0 & 0 & 0 & 0 & 0 & 0 & 0 & 0 & 0 & 1,25 & 0 & 0,63 \\
\hline FEC & 0 & 0 & 0 & 0 & 0 & 0 & 0 & 100 & 50 & 61,25 & 37,5 & 49,37 \\
\hline FCE & 0 & 0 & 0 & 0 & 0 & 0 & 0 & 0 & 0 & 0 & 0 & 0 \\
\hline EFC & 0 & 0 & 0 & 0 & 0 & 0 & 0 & 0 & 0 & 36,25 & 62,5 & 49,37 \\
\hline ECF & 0 & 0 & 0 & 0 & 0 & 0 & 0 & 0 & 0 & 1,25 & 0 & 0,63 \\
\hline
\end{tabular}

\begin{tabular}{|c|c|c|c|c|c|c|c|c|c|c|c|c|}
\hline \multicolumn{13}{|c|}{ Participante P12 } \\
\hline CEF & 100 & 100 & 100 & 100 & 100 & 100 & 0 & 0 & 0 & 0 & 0 & 0 \\
\hline CFE & 0 & 0 & 0 & 0 & 0 & 0 & 0 & 0 & 0 & 0 & 0 & 0 \\
\hline FEC & 0 & 0 & 0 & 0 & 0 & 0 & 100 & 100 & 100 & 100 & 100 & 100 \\
\hline FCE & 0 & 0 & 0 & 0 & 0 & 0 & 0 & 0 & 0 & 0 & 0 & 0 \\
\hline $\mathrm{EFC}$ & 0 & 0 & 0 & 0 & 0 & 0 & 0 & 0 & 0 & 0 & 0 & 0 \\
\hline ECF & 0 & 0 & 0 & 0 & 0 & 0 & 0 & 0 & 0 & 0 & 0 & 0 \\
\hline \multicolumn{13}{|c|}{ Participante P13 } \\
\hline CEF & 98,75 & 100 & 99,37 & 100 & 100 & 100 & 97,5 & 0 & 48,75 & 0 & 56,25 & 28,12 \\
\hline CFE & 1,25 & 0 & 0,63 & 0 & 0 & 0 & 0 & 0 & 0 & 0 & 0 & 0 \\
\hline FEC & 0 & 0 & 0 & 0 & 0 & 0 & 1,25 & 100 & 50,62 & 100 & 43,75 & 71,88 \\
\hline FCE & 0 & 0 & 0 & 0 & 0 & 0 & 0 & 0 & 0 & 0 & 0 & 0 \\
\hline $\mathrm{EFC}$ & 0 & 0 & 0 & 0 & 0 & 0 & 1,25 & 0 & 0,63 & 0 & 0 & 0 \\
\hline ECF & 0 & 0 & 0 & 0 & 0 & 0 & 0 & 0 & 0 & 0 & 0 & 0 \\
\hline \multicolumn{13}{|c|}{ Participante P14 } \\
\hline $\mathrm{CEF}$ & 100 & 100 & 100 & 100 & 95 & 97,5 & 98,75 & 100 & 99,37 & 100 & 100 & 99,37 \\
\hline CFE & 0 & 0 & 0 & 0 & 0 & 0 & 0 & 0 & 0 & 0 & 0 & 0,63 \\
\hline FEC & 0 & 0 & 0 & 0 & 5 & 2.5 & 0 & 0 & 0 & 0 & 0 & 0 \\
\hline FCE & 0 & 0 & 0 & 0 & 0 & 0 & 0 & 0 & 0 & 0 & 0 & 0 \\
\hline $\mathrm{EFC}$ & 0 & 0 & 0 & 0 & 0 & 0 & 1,25 & 0 & 0,63 & 0 & 0 & 0 \\
\hline ECF & 0 & 0 & 0 & 0 & 0 & 0 & 0 & 0 & 0 & 0 & 0 & 0 \\
\hline
\end{tabular}

Na Fase 4, quando a pergunta foi apresentada, apenas o Participante P12 iniciou essa fase respondendo corretamente, como pode ser observado na Figura 1. $\mathrm{Na}$ Tabela 1 observa-se que esse participante foi o único que respondeu corretamente na seqüência FEC, especificada pela pergunta, em $100 \%$ das tentativas dessa fase. Os outros três participantes (P11, P13 e P14) continuaram emitindo a sequiência CEF (previamente especificada pela instrução correspondente na Fase 2) em mais de $97 \%$ das tentativas da primeira sessão da Fase 4, apesar do seguimento de instrução não mais produzir pontos. No início da segunda sessão da Fase 4, quando a pergunta foi reapresentada, os Participantes P11 e P13 passaram a responder corretamente, emitindo a seqüência especificada pela pergunta (FEC) em 100\% das tentativas dessa sessão. Já o Participante P14 continuou seguindo a instrução.

$\mathrm{Na}$ Fase 5, quando as contingências foram alteradas e o comportamento especificado pela pergunta deixou de produzir pontos, nenhum dos quatro participantes respondeu corretamente, de acordo com as novas contingências de reforço, como pode ser obser- vado na Figura 1. Na Tabela 1 observa-se que dois participantes (P12 e P14) continuaram apresentando o mesmo desempenho apresentado na Fase 4. O Participante $\mathrm{P} 12$ continuou respondendo de acordo com a pergunta, previamente apresentada na Fase 4, e o Participante P14 continuou respondendo de acordo com a instrução correspondente, previamente apresentada na Fase 2. Os outros dois participantes (P11 e P13), na primeira sessão da Fase 5, responderam na seqüência FEC (previamente especificada pela pergunta na Fase 4) em $61.25 \%$ e $100 \%$ das tentativas, respectivamente. $\mathrm{Na}$ segunda sessão esses dois participantes mudaram os seus desempenhos e passaram a emitir as sequiências EFC e CEF em $62.5 \%$ e $56.25 \%$ das tentativas, respectivamente.

\section{Condição II (PI)}

A Figura 2 mostra a frequiência acumulada de sequiências de respostas corretas e incorretas, emitidas por cada participante da Condição II (PI), durante as Fases 2, 3, 4 e 5. A Tabela 2 mostra as porcentagens de sequiências de respostas apresentadas durante as 
Fases 2, 3, 4 e 5, pelos participantes da Condição II (PI).
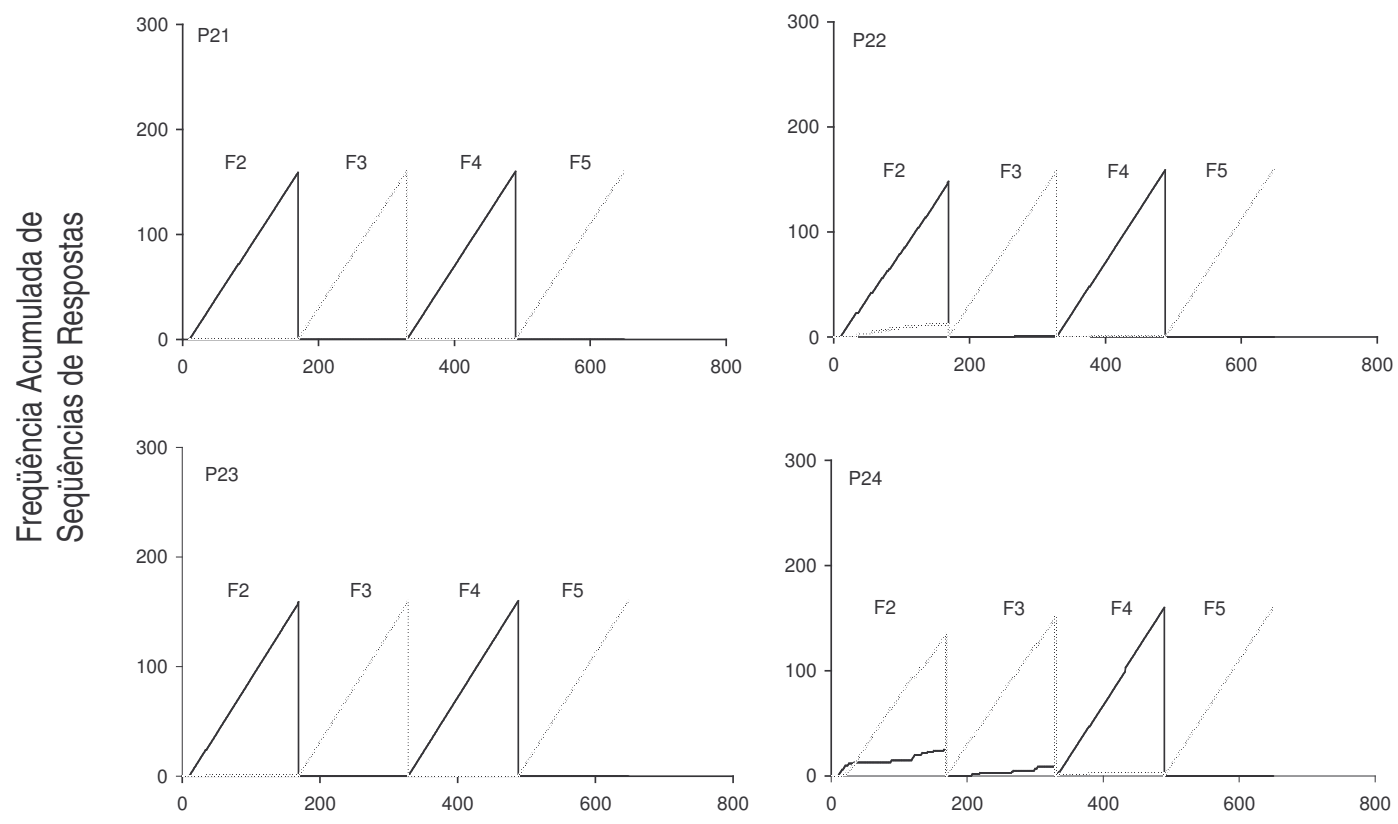

Tentativas

Figura 2: Frequiência acumulada de sequiências de respostas corretas (linha sólida preta) e incorretas (linha tracejada cinza), para cada participante $(\mathrm{P})$, durante cada fase $(\mathrm{F})$ experimental na Condição II (PI). Quebras na curva acumulada indicam mudanças de fase.

Na Fase 2 da Condição II (PI), quando a pergunta foi introduzida, pode-se observar na Figura 2 que todos os participantes dessa condição (P21, P22, P23 e P24) iniciaram esta fase respondendo corretamente. Na Tabela 2 observa-se que três participantes (P21, P22 e P23) responderam corretamente na sequiência
CEF, especificada pela pergunta, em mais de $92 \%$ das tentativas dessa fase. O Participante P24 variou bastante o seu desempenho e, desse modo, emitiu a seqüência correta (CEF) em apenas $15 \%$ das tentativas dessa fase.

Tabela 2: Porcentagens de Seqüências de Respostas (Seq.) Emitidas pelos Participantes P21, P22, P23 e P24 da Condição II, Durante a Primeira e a Segunda sessão de cada Fase, e Durante toda a Fase Experimental, nas Fases 2, 3, 4 e 5

\begin{tabular}{|c|c|c|c|c|c|c|c|c|c|c|c|c|}
\hline & \multicolumn{3}{|c|}{ Fase 2} & \multicolumn{3}{|c|}{ Fase 3} & \multicolumn{3}{|c|}{ Fase 4} & \multicolumn{3}{|c|}{ Fase 5} \\
\hline & Primeira & Segunda & Durante & Primeira & Segunda & Durante & Primeira & Segunda & Durante & Primeira & Segunda & Durante \\
\hline \multicolumn{13}{|l|}{ Seq. } \\
\hline \multicolumn{13}{|c|}{ Participante P21 } \\
\hline $\mathrm{CEF}$ & 100 & 100 & 100 & 98,75 & 100 & 99,37 & 0 & 0 & 0 & 0 & 0 & 0 \\
\hline $\mathrm{CFE}$ & 0 & 0 & 0 & 0 & 0 & 0 & 0 & 0 & 0 & 0 & 0 & 0 \\
\hline FEC & 0 & 0 & 0 & 0 & 0 & 0 & 100 & 100 & 100 & 100 & 100 & 100 \\
\hline FCE & 0 & 0 & 0 & 0 & 0 & 0 & 0 & 0 & 0 & 0 & 0 & 0 \\
\hline $\mathrm{EFC}$ & 0 & 0 & 0 & 0 & 0 & 0 & 0 & 0 & 0 & 0 & 0 & 0 \\
\hline $\mathrm{ECF}$ & 0 & 0 & 0 & 1,25 & 0 & 0,63 & 0 & 0 & 0 & 0 & 0 & 0 \\
\hline \multicolumn{13}{|c|}{ Participante P22 } \\
\hline $\mathrm{CEF}$ & 90 & 95 & 92,5 & 95 & 40 & 67,5 & 0 & 0 & 0 & 0 & 0 & 0 \\
\hline CFE & 1,25 & 1,25 & 1,25 & 0 & 3,75 & 1,88 & 0 & 0 & 0 & 0 & 0 & 0 \\
\hline FEC & 2,5 & 2,5 & 2,5 & & 0 & 0 & 98,75 & 100 & 99,37 & 100 & 100 & 100 \\
\hline FCE & 3,75 & 0 & 1,88 & 3,75 & 53,75 & 28,75 & 0 & 0 & 0 & 0 & 0 & 0 \\
\hline
\end{tabular}




\begin{tabular}{|c|c|c|c|c|c|c|c|c|c|c|c|c|}
\hline EFC & 2,5 & 1,25 & 1,88 & 0 & 1,25 & 0,63 & 1,25 & 0 & 0,63 & 0 & 0 & 0 \\
\hline ECF & 0 & 0 & 0 & 1,25 & 1,25 & 1,25 & 0 & 0 & 0 & 0 & 0 & $\begin{array}{l}0 \\
\text { cont. }\end{array}$ \\
\hline \multicolumn{13}{|c|}{ Participante P23 } \\
\hline CEF & 98,75 & 100 & 99,37 & 100 & 100 & 100 & 0 & 0 & 0 & 0 & 0 & 0 \\
\hline CFE & 1,25 & 0 & 0,63 & 0 & 0 & 0 & 0 & 0 & 0 & 0 & 0 & 0 \\
\hline FEC & 0 & 0 & 0 & 0 & 0 & 0 & 100 & 100 & 100 & 100 & 100 & 100 \\
\hline FCE & 0 & 0 & 0 & 0 & 0 & 0 & 0 & 0 & 0 & 0 & 0 & 0 \\
\hline $\mathrm{EFC}$ & 0 & 0 & 0 & 0 & 0 & 0 & 0 & 0 & 0 & 0 & 0 & 0 \\
\hline ECF & 0 & 0 & 0 & 0 & 0 & 0 & 0 & 0 & 0 & 0 & 0 & 0 \\
\hline \multicolumn{13}{|c|}{ Participante P24 } \\
\hline CEF & 18,75 & 11,25 & 15 & 11,25 & 16,25 & 13,75 & 0 & 0 & 0 & 0 & 0 & 0 \\
\hline CFE & 35 & 27,5 & 31,25 & 25 & 18,75 & 21,88 & 0 & 0 & 0 & 0 & 0 & 0 \\
\hline FEC & 15 & 21,25 & 18,13 & 30 & 18,75 & 24,38 & 96,25 & 100 & 98,12 & 100 & 100 & 100 \\
\hline FCE & 25 & 17,5 & 21,25 & 6,25 & 12,5 & 9,38 & 2,5 & 0 & 1,25 & 0 & 0 & 0 \\
\hline EFC & 3,75 & 7,5 & 5,63 & 21,25 & 13,75 & 17,5 & 1,25 & 0 & 0,63 & 0 & 0 & 0 \\
\hline ECF & 2,5 & 15 & 8,75 & 6,25 & 20 & 13,12 & 0 & 0 & 0 & 0 & 0 & 0 \\
\hline
\end{tabular}

$\mathrm{Na}$ Fase 3, quando as contingências foram alteradas e o comportamento especificado pela pergunta deixou de produzir pontos, nenhum desses quatro participantes (P11, P12, P13 e P14) respondeu corretamente, de acordo com as novas contingências de reforço, como pode ser observado na Figura 2. Na Tabela 2 observa-se que dois participantes (P21 e P23) continuaram respondendo na seqüência CEF (previamente especificada pela pergunta na Fase 2) em mais de $99 \%$ das tentativas dessa fase. O Participante P22, durante a primeira sessão, também continuou emitindo a sequiência CEF, mas a partir da segunda sessão passou a variar seu desempenho. Já o Participante P24 continuou variando o seu desempenho, apresentando diferentes sequiências de respostas, chegando inclusive a emitir a seqüência EFC (seqüência correta na Fase 3) em 17.5\% das tentativas.

Na Fase 4, quando a instrução correspondente foi apresentada, pode-se observar na Figura 2 que todos os participantes responderam corretamente. Na Tabela 2 observa-se que esses participantes fizeram isso em mais de $98 \%$ das tentativas dessa fase.

Na Fase 5, quando as contingências foram alteradas e o seguimento de instrução deixou de produzir pontos, todos os quatro participantes continuaram respondendo na seqüência FEC (previamente especificada pela instrução correspondente na Fase 4) durante toda a Fase 5, como pode ser observado na Tabela 2 .

\section{DISCUSSÃO}

$\mathrm{Na}$ linha de pesquisa que investiga o controle por regras, alguns resultados experimentais têm sugerido que, quando o comportamento é inicialmente estabelecido por regras ele tende a permanecer inalterado quando as contingências de reforço mudam (Paracampo \& cols., 2001; Shimoff, Catania \& Matthews, 1981).
Os resultados das Fases 2 e 3 da Condição I (IP) do presente estudo, mostrando que todos os quatro participantes dessa condição seguiram a instrução correspondente na Fase 2 e que o comportamento de seguir essa instrução não mudou acompanhando as mudanças nas contingências na Fase 3, apóiam essa sugestão. Além disso, os resultados das Fases 2 e 3 de três (P21, P22 e P23) dos quatro participantes da Condição II (PI) do presente estudo, sugerem que perguntas que especificam o comportamento que produz reforço, tal como instruções que especificam esse comportamento, também podem estabelecer comportamentos novos (no caso do presente estudo, a emissão de uma sequiência de três diferentes respostas por seis vezes consecutivas) e gerar insensibilidade do comportamento a mudanças nas contingências de reforço programadas. Os resultados das Fases 2 e 3 desses sete participantes (P11, P12, P13, P14, P21, P22 e P23) sugerem, portanto, que estímulos antecedentes verbais que especificam o comportamento que produz reforço podem funcionar como regras, independentemente de se tais estímulos são apresentados na forma de instruções ou de perguntas.

Contudo, os resultados da primeira sessão da Fase 4, mostrando que apenas um dos quatro participantes da Condição I (IP) respondeu tal como especificado pela pergunta, enquanto todos os quatro participantes da Condição II (PI) responderam de acordo com a instrução, sugerem que nem sempre perguntas que especificam o comportamento que produz reforço funcionam como regras. Sugerem também que não basta que um estímulo antecedente verbal descreva o comportamento a ser emitido para que esse comportamento ocorra.

Além disso, os resultados das Fases 2, 3, 4 e 5 das duas condições sugerem que o controle exercido por uma história de reforço para o comportamento de seguir instruções tende a impedir o controle subse- 
qüente por perguntas sobre o comportamento por elas especificado, enquanto que o controle exercido por uma história prévia de reforço para o comportamento especificado por uma pergunta tende a não interferir no seguimento subseqüiente de instruções. Sugerem ainda que perguntas tendem a gerar um desempenho mais variável que instruções.

Em síntese, essa análise sugere que, diferente de perguntas que não especificam o comportamento que produz reforço (Silva \& Albuquerque, submetido), perguntas que especificam esse comportamento, tal como instruções, podem ser classificadas como regras. Mas o controle exercido por instruções tende a ser mais forte quando comparado com o controle exercido por perguntas. Ou seja, o comportamento que se segue à apresentação de uma instrução tende a apresentar pouca variação em relação ao comportamento especificado na própria instrução e a ser mais persistente quando comparado com o comportamento que se segue à apresentação de uma pergunta. Pesquisas futuras que comparassem, em um mesmo ouvinte, os efeitos de perguntas e instruções que especificam e que não o comportamento que produz reforço poderiam contribuir para esclarecer se regras deveriam ser definidas como estímulos antecedentes verbais especificadores de contingências (Skinner, 1969) ou como estímulos antecedentes verbais (Hayes, 1986). Ou seja, poderiam contribuir para esclarecer se regras deveriam ser definidas também com base em suas propriedades formais.

\section{REFERÊNCIAS}

Albuquerque, L. C. \& Ferreira, K. V. D. (2001). Efeitos de regras com diferentes extensões sobre o comportamento humano. Psicologia: Reflexão e Crítica, 14, 143-155.

Albuquerque, L. C., Matos, M. A., Souza, D. G. de \& Paracampo, C. C. P. (2004). Investigação do controle por regras e do controle por histórias de reforço sobre o comportamento humano. Psicologia: Reflexão e Crítica, 17, 395-412.

Bentall, R. P. \& Lowe, C. F. (1987). The role of verbal behavior in human learning III. Instructional effects on children. Journal of the Experimental Analysis of Behavior, 47, 177-190.

Catania, A. C. (1998). Learning (4 ${ }^{\mathrm{a}}$ ed.). New Jersey: Prentice Hall.

Catania, A. C.; Matthews, A. \& Shimoff, E. (1982). Instructed versus shaped human verbal behavior: interactions with nonverbal responding. Journal of the Experimental Analysis of Behavior, 38, 233-248.

Catania, A. C.; Shimoff, E. \& Matthews, A. (1989). An experimental analysis of rule-governed behavior. Em S. C. Hayes (Org.), Rule-governed behavior: cognition, contingencies, and instructional control (pp. 119-150). New York: Plenum.

Cerutti, D. T. (1991). Discriminative versus reinforcing properties of schedules as determinants of schedule insensitivity in humans. The Psychological Record, 41, 51-67.

Dixon, M. R. \& Hayes, L. J. (1998). Effects of differing instructional histories on the resurgence of rule-following. The Psychological Record, 48, 275-292.
Harzem, P., Lowe, C. F. \& Bagshaw, M. (1978). Verbal control in human operant behavior. Psychological Record, 28, 405-423.

Hayes, S. C. (1986) The case of the silent dog-verbal reports and the analysis of rules: a review of Ericsson and Simon's "Protocol Analysis: verbal reports as data." Journal of Experimental Analysis of Behavior, 45, 351-363.

Hayes, S. C. \& Hayes, L. J. (1989). The verbal action of the listener as a basis for rule-governance. Em S. C. Hayes (Org.), Rule governed behavior: cognition, contingencies, and instructional control (pp. 153-190). New York: Plenum.

LeFrancois, J. R.; Chase, P. N. \& Joyce, J. (1988). The effects of variety of instructions on human fixed-interval performance. Journal of the Experimental Analysis of Behavior, 49, 383-393.

Lippman, L. G. \& Meyer, M. E. (1967). Fixed interval performance as related instructions and to subjects verbalizations of the contingency. Psychonomic Science, 8, 135-136.

Lowe, C. F. (1979). Determinants of human operant behaviour. Em M. D. Zeiler \& P. Harzem (Eds.), Advances in analysis of behaviour: Vol. 1 Reinforcement and the organization of behaviour (pp. 159-192). Chichester, England: Wiley.

Paracampo, C. C. P., de Souza, D. G., Matos, M. A. \& Albuquerque, L. C. (2001). Efeitos de mudanças em contingências de reforço sobre o comportamento verbal e o não-verbal. Acta Comportamentalia. 9, 31-55.

Pouthas, V., Droit, S., Jacquet, Y. \& Wearden, J. H. (1990). Temporal differentiation of response duration in children of different ages: developmental changes in relations between verbal and nonverbal behavior. Journal of the Experimental Analysis of Behavior, 53, 21-31.

Rosenfarb, I. S., Newland, M. C., Brannon, S. E. \& Howey, D. S. (1992). Effects of self-generated rules on the development of schedule-controlled behavior. Journal of the Experimental Analysis of Behavior, 58, 107-121.

Shimoff, E.; Catania, A. C. \& Matthews B. A. (1981). Uninstructed human responding: Sensitivity of low-rate performance to schedule contingencies. Journal of the Experimental Analysis of Behavior, 36, 207-220.

Silva, F. M. \& Albuquerque, L. C. (submetido). Efeitos de perguntas e de histórias experimentais sobre o seguir regras. Psicologia: Teoria e Pesquisa.

Simonassi, L., Oliveira, C. \& Gosch, C. (1997). Exposição a contingências, conteúdo de instrução e formulação de regras. Psicologia: Teoria e Pesquisa, 13, 189-195.

Skinner, B. F. (1957). Verbal behavior. Massachusetts: Prentice Hall.

Skinner, B. F. (1969). Contingencies of reinforcement: a theoretical analysis. New York: Appleton-Century-Crofts.

Torgrud, L. J. \& Holborn, S. W. (1990). The effects of verbal performance descriptions on nonverbal operant responding. Journal of the Experimental Analysis of Behavior, 54, 273-291.

Vaughan, M E. (1985). Repeated acquisition in the analysis of rule-governed behavior. Journal of the Experimental Analysis of Behavior, 44, 175-184.

Zettle, R. D. \& Hayes, S. C. (1982). Rule-governed behavior: a potential theoretical framework for cognitive-behavior therapy. Em P. C. Kendall (Ed.), Advances in cognitive-behavioral research and therapy (pp. 73-118). New York: Academic Press. 


\section{Notas:}

O presente trabalho contou com o auxílio do CNPq - Processo 403100/2003-8.

2 O termo sensibilidade está sendo usado para descrever o comportamento que está sob o controle de suas consequiências imediatas e o termo insensibilidade para descrever o comportamento que não está sob o controle de suas conseqüências imediatas em uma determinada situação particular.

3 Para evitar coincidência entre o comportamento descrito pela instrução correspondente ou pela pergunta no início da Fase 2 e o comportamento apresentado pelo participante na Fase 1, a instrução correspondente e a pergunta podiam especificar a sequiência alternativa descrita entre colchetes.

\section{Sobre os autores:}

Mariella Vasconcelos Nogueira Braga: Psicóloga e Doutoranda do Programa de Pós-Graduação em Teoria e Pesquisa do Comportamento da Universidade Federal do Pará. Bolsista de Doutorado da CAPES.

Luiz Carlos de Albuquerque: Professor do Departamento de Psicologia Experimental da Universidade Federal do Pará e do Programa de Pós-Graduação em Teoria e Pesquisa do Comportamento da mesma Universidade. Endereço eletrônico: lca@cpgp.ufpa.br.

Carla Cristina Paiva Paracampo: Professora do Departamento de Psicologia Experimental da Universidade Federal do Pará e do Programa de Pós-Graduação em Teoria e Pesquisa do Comportamento da mesma Universidade. 
